\author{
第1日・9月13日(月) $9: 30 \sim 11 ： 30$ J会場
}

学術奨励賞

候補論文

( 基礎系)

1

\section{Mechanosensitivity of GIRK Channels Is Mediated by Protein Kinase C-dependent Channel-Phosphatidylinositol 4,5-Bisphosphate Interaction}

\author{
Liyan Zhang, Jong-Kook Lee, Scott A. John, Nobuyuki Uozumi, Itsuo Kodama \\ Department of Circulation, Research Institute of Environmental Medicine, \\ Bioscience and Biotechnology Center, Nagoya University, Nagoya.
}

Gprotein-activated inwardly rectifying $\mathrm{K}^{+}$channel (GIRK or Kir3) currents are inhibited by mechanical stretch of the cell membrane, but the underlying mechanisms are not understood. In Xenopus oocytes heterologously expressing GIRK channels, membrane stretch induced by $50 \%$ reduction of osmotic pressure caused a prompt reduction of GIRK1/4, GIRK1, and GIRK4 currents by $16.6-42.6 \%$. Comparable GIRK current reduction was produced by protein kinase C (PKC) activation (phorbol 12myristate 13-acetate). The mechanosensitivity of the GIRK4 current was abolished by pretreatment with PKC inhibitors (staurosporine or calphostin C). Neither hypo-osmotic challenge nor PKC activation affected IRK1 currents. GIRK4 chimera (GIRK4-IRK1- $\left(\operatorname{Lys}^{300}-\operatorname{Leu}^{2+45}\right)$ ) and single point mutant (GIRK4 (I229L)), in which the phosphatidylinositol 4,5-bisphosphate (PIP.) binding domain or residue was replaced by the corresponding region of IRK1 to strengthen the channel-PIP 2 interaction, showed no mechanosensitivity and minimal PKC sensitivity. IRK1 gained mechanosensitivity and PKC sensitivity by reverse double point mutation of the $\mathrm{PIP}_{2}$ binding domain (L222I/R213Q). Overexpression of G $\beta \gamma$, which is known to strengthen the channel-PIP 2 interaction, attenuated the mechanosensitivity of GIRK4 channels. In oocytes expressing a pleckstrin homology domain of PLC- $\delta$ tagged with green fluorescent protein, hypo-osmotic challenge or PKC activation caused a translocation of the fluorescence signal from the cell membrane to the cytosol, reflecting $\mathrm{PIP}_{2}$ hydrolysis. The translocation was prevented by pretreatment with PKC inhibitors. Involvement of PKC activation in the mechanosensitivity of muscarinic $\mathrm{K}^{+}$channels was confirmed in native rabbit atrial myocytes. These results suggest that the mechanosensitivity of GIRK channels is mediated primarily by channel-PIP, interaction, with PKC playing an important role in modulating the interaction probably through PIP. hydrolysis. (J Biol Chem, $2004 ; 8: 7037 \sim 7047)$ 
学術奖励賞

候補論文

(基礎系)

2

\title{
Mechanism of ST Elevation and Ventricular Arrhythmias in an Experimental Brugada Syndrome Model
}

\author{
Masaomi Kimura, Takao Kobayashi, Shingen Owada, Keiichi Ashikaga, Takumi \\ Higuma, Shingo Sasaki, Atsushi Iwasa, Shigeru Motomura, Ken Okumura \\ Second Department of Internal Medicine and Department of Pharmacology, \\ Hirosaki University School of Medicine, Hirosaki, Japan.
}

Background : Although phase 2 reentry is said to be responsible for initiation of ventricular tachycardia (VT) in Brugada syndrome, information about the activation sequence during VT is limited.

Methods and Results: We developed an experimental Brugada syndrome model using a canine isolated right ventricular preparation cross-circulated with arterial blood of a supporter dog and examined the VT mechanism. Two plaque electrodes $(35 \times 30 \mathrm{~mm})$ containing 96 bipolar electrodes were attached to the endocardium and epicardium. Saddleback and coved types of ST elevation in transmural ECG were induced by pilsicainide, a pure sodium channel blocker, and pinacidil, a $K_{\Lambda \mathrm{TP}}$ channel opener. Eighteen polymorphic VT episodes were recorded in 9 of the 12 preparations associated with ST elevation. Fourteen episodes spontaneously developed in 5 preparations after an extrasystole during basic drive pacing. Analysis of local recovery times revealed increased dispersion especially in epicardium, and the extrasystole originated from a site with a short recovery time, suggesting that phase 2 reentry was its mechanism. The other 4 VTs in 4 preparations were induced by premature stimulation. Analysis of the activation sequences during VT revealed reentry between epicardium and endocardium or reentry around an arc of a functional block confined to epicardium or endocardium with bystander activation of the other.

Conclusions : Electrical heterogeneity in the recovery phase was induced in this experimental Brugada syndrome model, which can be a substrate for the development of phase 2 reentry and the subsequent reentry around an arc of the functional block, resulting in sustained VT. (Circulation, $2004 ; 109: 125$ 131) 


\title{
Identifying Patterns of Spatial Current Dispersion that Characterise and Separate the Brugada Syndrome and Complete Right-Bundle Branch Block
} 候補論文

\author{
Kandori A, Shimizu W, Yokokawa M, Noda T, Kamakura S, Miyatake K, \\ Murakami M, Miyashita T, Ogata K. Tsukada K \\ Central Research Laboratory, Hitachi, Ltd, Tokyo, Japan.
}

The aim of the study was to detect patterns of spatial - current distribution in the late QRS and early ST-segments that distinguish Brugada-syndrome cases from complete right-bundle branch block (CRBBB). Magnetocardiograms (MCGs) were recorded from Brugada-syndrome patients $(n=6)$, CRBBB patients $(n=4)$ and the members of a control group $(n=33)$. The current distributions at six time points from $\mathrm{Q}$ - onset were estimated by producing current-arrow maps (CAMs). The angle of the current arrow of maximum amplitude at each time point was calculated. In the Brugada cases, the characteristic ST elevation was seen above the upper right chest. and abnormal currents appeared to be present in the right-ventricular outflow tract (RVOT). The angles of the abnormal arrows were -78 degrees $+/-$ 51 degrees at $100 \mathrm{~ms}$ and -50 degrees $+/-61$ degrees at $110 \mathrm{~ms}$. In the cases of CRBBB, wide S- and $\mathrm{R}$-waves were recorded above the upper right and lower right chest. respectively. The angles of the abnormal arrows for CRBBB were 152 degrees $+/-19$ degrees at $100 \mathrm{~ms}, 159$ degrees $+/-20$ degrees at $110 \mathrm{~ms}$, and 157 degrees +/- 19 degrees at $120 \mathrm{~ms}$. The findings suggest that an abnormal current from the RVOT to the upper left chest may be a feature of the Brugada syndrome, and that the direction of this current is completely different from that seen in CRBBB. (Med Biol Eng Comput, 2004 : $42: 236 \sim 244)$ 
4

\author{
S. Yamada', K. Tsukada ${ }^{2}$, T. Miyashita ${ }^{2}$, K. Kuga', I. Yamaguchi ${ }^{1}$ \\ ${ }^{1}$ Institute of Clinical Medicine, University of Tsukuba, Tsukuba, Japan \\ ${ }^{2}$ Hitachi Central Research Laboratory, Hitachi Ltd., Kokubunji, Japan
}

\begin{abstract}
Aims : We analysed the cardiac magnetic fields on the body surface to visualize electrical currents noninvasively during reentrant arrhythmias.

Methods and results : Seven patients with counterclockwise atrial flutter (AFL) were studied during 17 episodes of AFL using 64-channel magnetocardiograms (MCGs) and electrophysiological study. Eight of the episodes were paroxysmal AFL, in which MCGs were recorded from the time of spontaneous onset to the time of termination. We constructed iso-magnetic field maps of the tangential components and produced MCG animations. With respect to AFL initiation, an atrial premature complex induced AFL. Prior to the initiation of AFL, atrial fibrillation (AF) transiently occurred. The cardiac magnetic fields revealed a single peak during sinus rhythm or with premature complexes but a disorganized pattern during AF. When AF transformed to AFL, the magnetic fields changed from a disorganized pattern to a single peak at first and then evolved to a circular pattern. During persistent AFL, the magnetic source moved in a counterclockwise circuit.

Conclusion : MCG animation can be used to visualize the sequence in which a premature complex transforms sinus rhythm to AFL via AF. Our findings indicate that MCGs can be used to identify noninvasively the mechanisms responsible for atrial tachyarrhythmias. (Europace, $2003 ; 5: 343 \sim 350$ )
\end{abstract}

